\title{
Identification of Clinical Indicators of Opioid Overdose using innovative EMS software analytics.
}

\author{
Silvia R. Verdugo ${ }^{1}$, Pam Farber ${ }^{1}$, John Selters ${ }^{1}$, Todd Stout $^{1}$, Karla D. Wagner ${ }^{2}$ \\ ${ }^{1}$ FirstWatch Solutions Inc., Carlsbad, California, United States, ${ }^{2}$ University of Nevada, Reno, Reno, Nevada, United States \\ Objective
}

To develop a set of clinical indicators of opioid overdose using Emergency Medical Services (EMS) records that included data from Computer Aided Dispatch (CAD), ProQA systems, Electronic Patient Care Reporting (ePCR) and Hospital Medical Records.

Introduction

In North America we experience the highest rate of drug related mortality in the world. In the US, overdose is now the leading cause of death among adults under 50. Each day more than 115 people in the United States die due to an opioid overdose. The opioid overdose national crisis is rapidly evolving due to changes in drug availability and the presence of adulterated fentanyl in some areas leading to a critical need for innovative methods to identify opioid overdoses for both surveillance and intervention purposes. As an effort to strengthen our understanding of the epidemic through surveillance of Emergency Medical Services (EMS) we have developed a set of clinical indicators that identify opioid overdose within the information provided by an Electronic Patient Care Reporting (ePCR), Computer Aided Dispatch (CAD), ProQA systems and Hospital Medical Records.

\section{Methods}

We initially created a set of EMS agency specific opioid overdose filters using FirstWatch ${ }^{\circledR}$ software as part of a public health research study. Following that initial development, we have built a generic set of opioid overdose identifiers. In the initial approach we used a Zoll Data System software for ePCR and TriTech Inform CAD to define 3 set of identifiers: (T1) captured calls in which naloxone was administered and a positive clinical response was documented, (T2) had the same criteria as T1 except there was no positive response to the administration of naloxone, and (T3) consisted of calls in which one or more drug-related keywords were present within the narrative of the ePCR. Because the initial analysis was conducted in the context of a single research study, we aimed to create a more generalizable set of identifiers of opioid overdose that would function across different EMS agencies, software, and data sources. In addition, we included variables provided by Hospital Medical Records to our filtering criteria to provide a more robust and complete set of opioid overdose clinical indicators.

\section{Results}

Utilizing the EMS data sources CAD, ProQA and ePCR as well as Hospital Medical Records we have developed a set of identifiers of opioid overdose. Utilizing FirstWatch ${ }^{\circledR}$ software analytics the following variables where coded into the software: 1 . CAD Data.Chief Complaint and Opioid Overdose Keyword search; 2. ProQA.- Protocols 6, 9, 23, 31 and 32; 3. ePCR.- Primary and Secondary Impressions, Chief Complaint, Intervention of Narcan (Naloxone) Administration, Vital Signs and Opioid Overdose Keyword search; 4. Medical Records.- Patient's Admission and Discharge Diagnosis (Diagram 1). The clinical indicators obtained from this analysis where created to be utilized across different EMS specific software vendors for CAD, ProQA and ePCR systems. For the Medical Records variables a single software vendor was available to be integrated into the analysis. Nonetheless, as we used the International Statistical Classification of Diseases and Related Health Problems codes on their 10th revision (ICD-10) our determining variable codes could be generalized to other Hospital Record system if they would become available.

\section{Conclusions}

Correctly identifying an opioid overdose can a be a challenge. Its clinical features are non-specific and bystanders fear repercussions of disclosing the nature of the 911 call. Determining the correct number of opioid overdoses requires a tailored identification process. A combination of clinical determinants and incorporation of multiple EMS data sources appears to be feasible in determining opioid overdose related 911 calls.

SDS Annual Conference Proceedings 2019. This is an Open Access article distributed under the terms of the Creative Commons AttributionNoncommercial 4.0 Unported License (http://creativecommons.org/licenses/by-nc/3.0/), permitting all non-commercial use, distribution, and reproduction in any medium, provided the original work is properly cited. 


\section{Acknowledgement}

The initial public health research study conducted to develop clinical indicators of overdose described as T1, T2 and T3 was supported by grants from the National Institute of General Medical Sciences (GM103440) and the National Institute on Drug Abuse (R01DA040648) and was approved by the UNR Institutional Review Board.

\section{References}

1. The United Nations Office on Drugs and Crime (UNODC) '2017 World Drug Report'.

2. Hedegaard H, Warner M, Miniño AM. Drug overdose deaths in the United States, 1999-2016. NCHS Data Brief, no 294. Hyattsville, MD: National Center for Health Statistics. 2017.

3. Multiple Cause of Death 1999-2016 on CDC Wide-ranging Online Data for Epidemiologic Research (CDC WONDER). Atlanta, GA: CDC, National Center for Health Statistics. 2017.

4. CDC/NCHS, National Vital Statistics System, Mortality. CDC Wonder, Atlanta, GA: US Department of Health and Human Services, CDC; 2017.

Diagram 1: Data sources and fields used to create clinical indicators of Opioid Overdose.

Diagram 1: Data sources and fields used to create clinical indicators of Opioid Overdose.
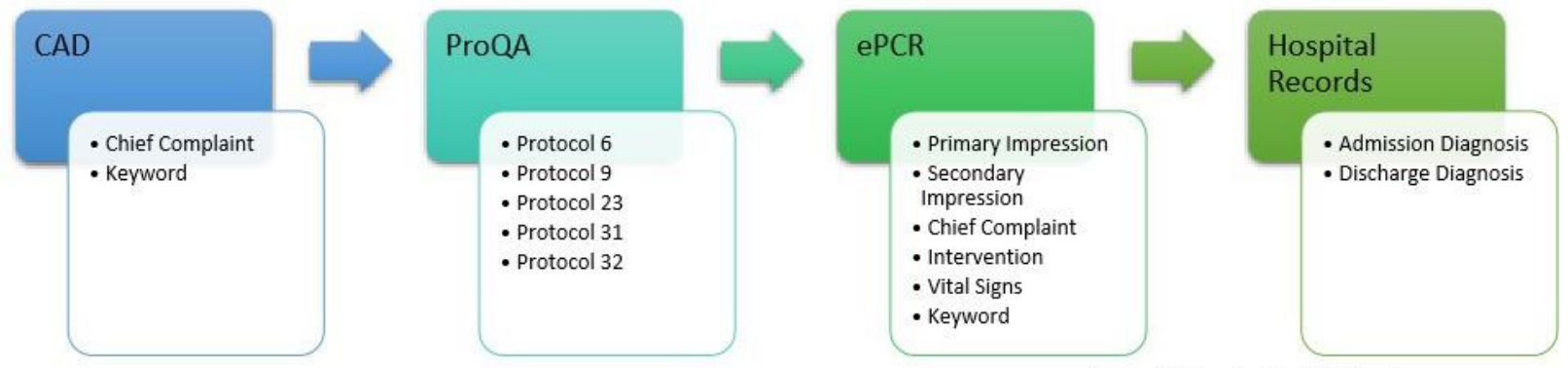

Data provided from FirstWatch" Solutions Inc. 